\title{
MONITOREO Y MEDICIÓN DEL AJUSTE DEL pH DEL AGUA TRATADA DEL RÍO CAUCA MEDIANTE ÍNDICES DE ESTABILIZACIÓN
}

\section{MONITORING AND MEASUREMENT BY STABILIZATION INDICES OF pH ADJUSTMENT OF TREATED WATER FROM THE CAUCA RIVER}

\author{
Karen Alejandra Bueno-Zabala ${ }^{1}$, Patricia Torres-Lozada ${ }^{2}$, Luis Germán Delgado-Cabrera ${ }^{3}$
}

\begin{abstract}
${ }^{1}$ Ingeniera Sanitaria, Magíster en Ingeniería. Grupo de Investigación Estudio y Control de la Contaminación Ambiental. Universidad del Valle, Cali, Colombia, Calle 13 \# 100 - 00, e-mail: karen.bueno@correounivalle.edu.co; ${ }^{2}$ Ingeniera Sanitaria, Universidad del Valle, Cali - Colombia. Magíster y Doctora en Ingeniería Civil, énfasis Hidráulica y Saneamiento, Profesora Titular, Grupo de Investigación Estudio y Control de la Contaminación Ambiental. Universidad del Valle, Cali - Colombia, Calle 13 \# 100 - 00, e-mail: patricia.torres@correounivalle.edu.co; ${ }^{3}$ Ingeniero Sanitario, Universidad del Valle, Cali - Colombia. Asesor Independiente - EMCALI EICE ESP. Cali - Colombia, Calle 4 \# 35 - 50, e-mail: lgdelca@gmail.com
\end{abstract}

Rev. U.D.C.A Act. \& Div. Cient. 17(2): 563-575, Julio-Diciembre, 2014

\section{RESUMEN}

Los bajos niveles de alcalinidad de las fuentes de abastecimiento y la adición de productos químicos para la coagulación y la desinfección utilizados en la potabilización del agua, generan la necesidad de realizar procesos de ajuste del $\mathrm{pH}$, como última etapa del tratamiento, con el fin de evitar fenómenos de agresividad, de corrosión o de incrustación, en los Sistemas de Distribución del Agua potable - SDA. Este artículo presenta los resultados de la aplicación de diferentes índices de estabilización para el monitoreo y el control del proceso de ajuste del $\mathrm{pH}$ del agua tratada del río Cauca, del cual, se abastece el $80 \%$ de la ciudad de Cali. Los índices se calcularon, tanto con información histórica como con los datos de seis jornadas de caracterización del agua tratada, encontrándose que ésta tiende a ser insaturada y corrosiva, lo que indica la necesidad de ajustar las condiciones del último proceso, evaluar otros tipos de alcalinizantes y continuar promoviendo la renovación de tuberías, en el SDA de la ciudad.

Palabras clave: Agua potable, juste del pH, sistema carbonato, distribución de Agua - SDA, tratamiento de agua.

\section{SUMMARY}

Low alkalinity levels of the supply sources and the addition of chemical coagulation and disinfection products for water purification, generates the need of the $\mathrm{pH}$ adjustment processes as the final treatment stage, in order to avoid phenomena of aggressiveness, corrosion or incrustation in the drinking water distribution system - DWDS. This article presents the results of the a application of different stabilization indexes for monitoring and the measurement of the $\mathrm{pH}$ adjustment process of the treated water from the Cauca River, which supplies $80 \%$ of the city of Cali. The indexes were calculated using historical information as well as data from six characterizations of the treated water, finding that this water tends to be unsaturated and corrosive, which indicates the need to adjust the conditions of the latter process, evaluate other types of alkalizing products and continue promoting the renovation of pipes in the DWDS in the city of Cali.

Key words: Drinking water, $\mathrm{pH}$ adjustment, carbonate system, distribution system - DWDS, water treatment.

\section{INTRODUCCIÓN}

En el tratamiento químico del agua, la coagulación altera el equilibrio del sistema carbonato, tendiendo a disminuir el $\mathrm{pH}$, cuando el coagulante se diluye, debido a la hidrólisis que libera iones hidrógeno y reduce la alcalinidad. Igualmente, la desinfección con cloro reduce la alcalinidad y el $\mathrm{pH}$, por las propiedades ácido-base del cloro que, al hidrolizarse, forma el ácido hipocloroso, que se disocia en iones de hidrógeno (WHO, 2011). Estos fenómenos hacen necesario reajustar el 
balance químico del agua al final del tratamiento, para asegurar su estabilidad en el sistema de distribución del agua - SDA (Trejo Vázquez \& García Díaz, 2007; Edzwald, 2010).

El ajuste químico del $\mathrm{pH}$ permite reducir la capacidad corrosiva del agua y evitar las incrustaciones (Casey, 2009), siendo la solución más común, de fácil aplicación y de bajo costo, comparada con la rehabilitación de los Sistemas de Distribución de Agua - SDA y consiste en mantener una deposición controlada de la película de carbonato de calcio $\left(\mathrm{CaCO}_{3}\right)$, sobre la superficie de las tuberías (De Sousa et al. 2010).

Los métodos de monitoreo y de medición del proceso de ajuste del $\mathrm{pH}$ pueden ser directos o indirectos; los directos (inspección física, análisis químico y técnicas microscópicas) proporcionan información sobre la situación actual de la sección de tubería analizada, siendo necesario realizar varios análisis, que evalúen el estado del SDA; estos métodos requieren de inspección continua, al menos de 90 días, con personal capacitado, equipos especializados y análisis de laboratorio, que generan mayores costos en la evaluación del proceso de ajuste del pH (Schock, 2002).

Los métodos indirectos (pruebas hidráulicas, registro e informe de quejas de los consumidores e índices de estabilización-IE) son una alternativa más simple, económica y de mayor rapidez que, en su conjunto, pueden presentar un análisis preventivo del ajuste del pH (Schock, 2002; Trujillo et al. 2008). Los IE permiten efectuar un control rápido y fácil del proceso y estimar o predecir las posibles consecuencias de la calidad del agua tratada sobre el SDA (Furtado et al. 2011); sin embargo, muchos de ellos han sido mal aplicados, en particular, cuando no se comprenden los principios fundamentales por los que fueron desarrollados y no se reconocen sus limitaciones (Schock, 2002; Birnhack et al. 2011).

En el agua cruda del río Cauca, que es la fuente de abastecimiento de, aproximadamente, el $80 \%$ de la población caleña, predominan bajos niveles de alcalinidad total, lo que, junto con el uso de productos químicos, que también reducen el $\mathrm{pH}$ por el consumo de alcalinidad y los daños que se presentan en el SDA, determinan que el proceso de ajuste del pH sea uno de los aspectos críticos del Sistema de Abastecimiento de Agua Potable - SAAP de la ciudad de Santiago de Cali (Pérez-Vidal et al. 2012). El objetivo de este artículo es presentar los resultados de la determinación de diferentes IE en el agua tratada del río Cauca y, con base en estos resultados, plantear recomendaciones, que pueden ser útiles para el mejoramiento del proceso de ajuste del $\mathrm{pH}$.

\section{MATERIALES Y MÉTODOS}

La primera etapa del estudio fue la identificación de los IE de mayor aplicabilidad para el control del proceso, la cual, se realizó mediante una amplia revisión de literatura, consultando fuentes primarias y secundarias, libros, tesis y reportes nacionales e internacionales, relacionados con el proceso. Con esta información, se realizó un análisis comparativo, con base en diversos aspectos, como variables requeridas para el cálculo, ecuaciones necesarias para determinar las variables no calculadas de manera rutinaria en la operación de las plantas, interpretación de los resultados de cada índice, utilidad y limitaciones.

La identificación de los IE de mayor aplicabilidad para el seguimiento del proceso de ajuste del $\mathrm{pH}$ en los SDA permitió realizar la etapa dos del estudio, que consistió en determinar estos IE, tanto con información histórica (datos diarios de la calidad del agua tratada, periodo 2010-2011, disponible en EMCALI EICE ESP) como con los resultados de seis jornadas de muestreo de 5 horas. Para el manejo de los datos, se aplicó un análisis estadístico descriptivo de indicadores de tendencia central, como la media, mediana, mínimos, máximos y cuartiles.

Finalmente, con base en el desarrollo y el análisis de los resultados de las etapas anteriores, se identificaron opciones para controlar y evaluar el proceso de ajuste del $\mathrm{pH}$, lo que, finalmente, permitió proponer recomendaciones que podrían mejorarlo.

\section{RESULTADOS Y DISCUSIÓN}

La tabla 1 presenta una síntesis de los IE de mayor aplicabilidad, para el control del proceso de ajuste del $\mathrm{pH}$, detallando las principales características de cada índice y resaltando aspectos, como la interpretación, la utilidad y las limitaciones, además de otras características, como simplicidad, tiempo de respuesta e influencia del material.

El ISL e IFC indican si el agua se encuentra en equilibrio respecto a la formación de la película de $\mathrm{CaCO}_{3}$, siendo útiles, inicialmente, para proporcionar las condiciones de sobresaturación en un SDA y aplicados a aguas blandas que, normalmente, se derivan de las cuencas altas (concentraciones de alcalinidad y dureza < 50mg/L) (Gray, 2008; De Sousa et al. 2010; Birnhack et al. 2011).

Los resultados de estos dos IE deberían ser positivos, lo que indicaría que mantenerlos en el tiempo representaría un impacto en la resistencia a problemas, como la corrosión (Loewenthal et al. 2004; Imran et al. 2005). En muchos casos, el ISL se ha utilizado inadecuadamente como solución 
Tabla 1. Resumen de las características de los índices y métodos aplicados en la evaluación de ajuste del pH.

\begin{tabular}{|c|c|c|}
\hline ÍNDICE & ECUACIÓN & CARACTERÍSTICAS \\
\hline $\begin{array}{l}\begin{array}{c}\text { Índice de } \\
\text { Saturación de } \\
\text { Langelier } \\
\text { (ISL) }\end{array} \\
\text { ISL>0, } \\
\text { sobresaturada. } \\
\text { ISL=0, saturada } \\
\text { (en equilibrio). } \\
\text { ISL < 0, } \\
\text { insaturada. }\end{array}$ & 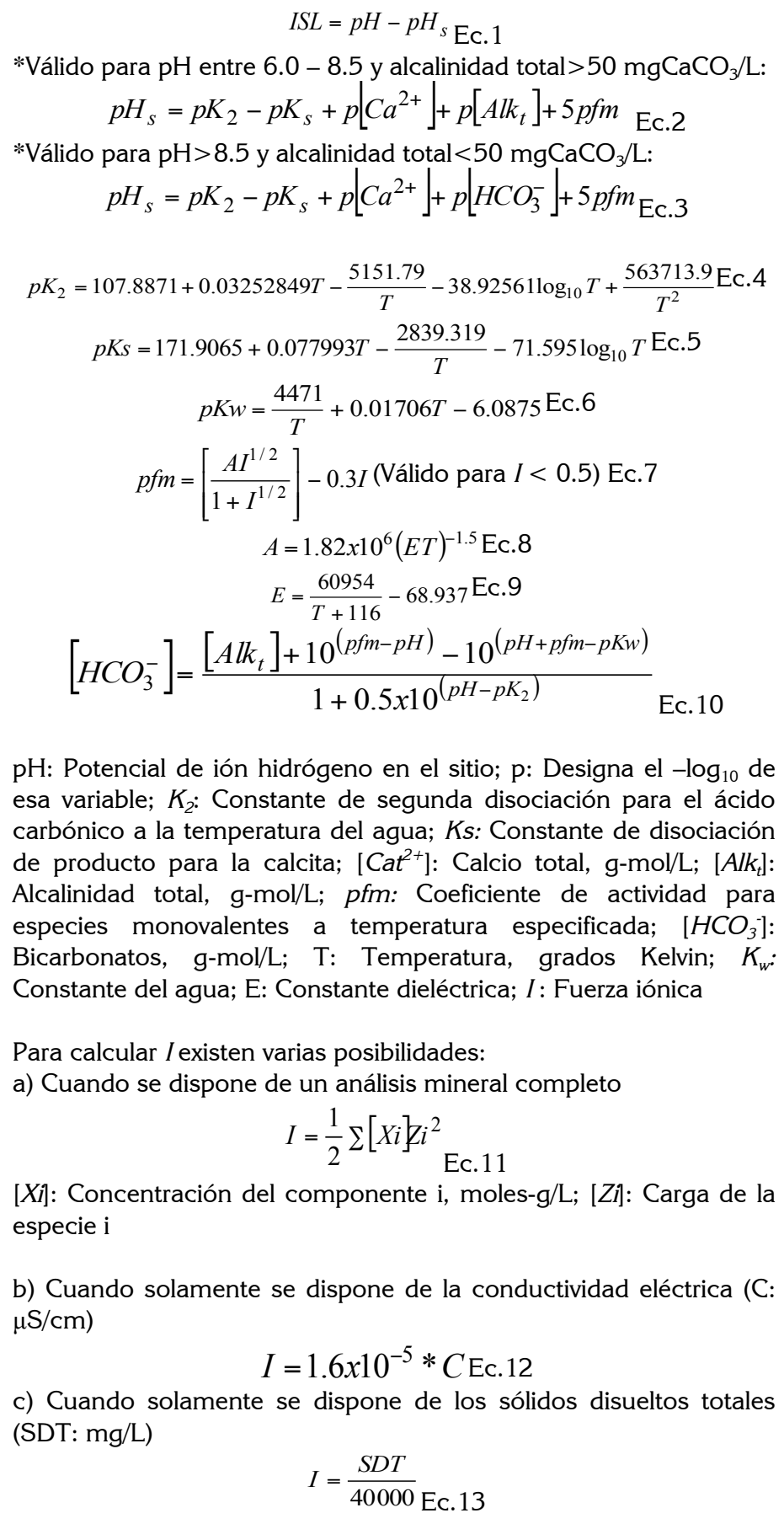 & $\begin{array}{l}\text { Utilidades: Indicador de } \\
\text { las condiciones de } \\
\text { precipitación del } \\
\text { carbonato de calcio. } \\
\text { Limitaciones: No } \\
\text { siempre es fiable en la } \\
\text { predicción de la } \\
\text { formación de la capa de } \\
\text { carbonato, porque } \\
\text { algunas aguas, con un } \\
\text { índice positivo, pueden } \\
\text { ser bastante corrosivas. } \\
\text { No es una forma } \\
\text { universal de control de la } \\
\text { corrosión. } \\
\text { No predice la cantidad de } \\
\text { CaCO }{ }_{3} \text { que precipitarán o } \\
\text { si su estructura } \\
\text { proporciona resistencia a } \\
\text { la corrosión. } \\
\text { La capacidad de } \\
\text { formación de la capa } \\
\text { protectora depende de } \\
\text { las propiedades del agua } \\
\text { a resistir cambios en el } \\
\text { valor del índice. }\end{array}$ \\
\hline $\begin{array}{l}\text { Índice de Fuerza } \\
\text { de conducción } \\
\text { de Mc Cauley } \\
\text { (IFC) } \\
\text { IFC }>1 \text {, agua }\end{array}$ & $\begin{array}{c}I F C=\frac{\mathrm{Ca}^{+2} * \mathrm{CO}_{3}^{-2}}{K S^{*} 10^{10}} \text { Ec. } 14 \\
\mathrm{CO}_{3}^{-2}=0.94 * \mathrm{Alc}_{\mathrm{HCO}_{3}^{-}} * 10^{\mathrm{pH-10}} \text { Ec. } 15\end{array}$ & $\begin{array}{l}\text { Utilidades: Mide la } \\
\text { tendencia de deposición } \\
\text { de la película de } \\
\text { carbonato de calcio en el } \\
\text { SDA. }\end{array}$ \\
\hline
\end{tabular}


Continuación tabla 1 .

\begin{tabular}{|c|c|c|}
\hline ÍNDICE & ECUACIÓN & CARACTERÍSTICAS \\
\hline $\begin{array}{l}\text { sobresaturada. } \\
\text { IFC }=1 \text {, agua } \\
\text { saturada (en } \\
\text { equilibrio). } \\
\text { IFC }<1 \text {, agua } \\
\text { insaturada. }\end{array}$ & 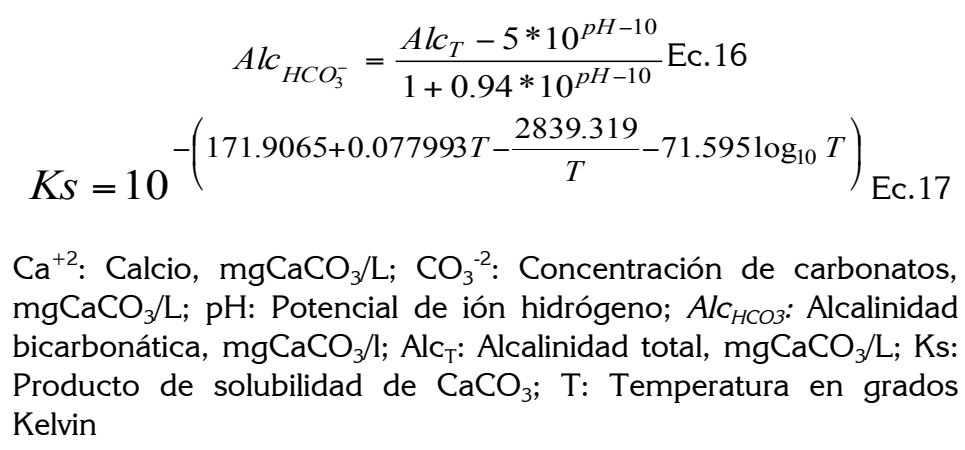 & $\begin{array}{l}\text { Limitaciones: No } \\
\text { implica directamente } \\
\text { efectos relacionados con } \\
\text { la corrosión, los cuales, } \\
\text { se deben reportar por } \\
\text { aparte. }\end{array}$ \\
\hline $\begin{array}{l}\text { Potencial de } \\
\text { Precipitación de } \\
\text { Carbonato de } \\
\text { Calcio (PPCC) } \\
\text { PPCC }>0 \text {, denota } \\
\text { sobresaturación y } \\
\text { los mgCaCO } \mathrm{m}_{3} / \mathrm{L} \\
\text { que deben } \\
\text { precipitar. } \\
\text { PPCC }<0 \text {, indica } \\
\text { insaturación y } \\
\text { cuanto CaCO } \\
\text { debe disolverse. }\end{array}$ & 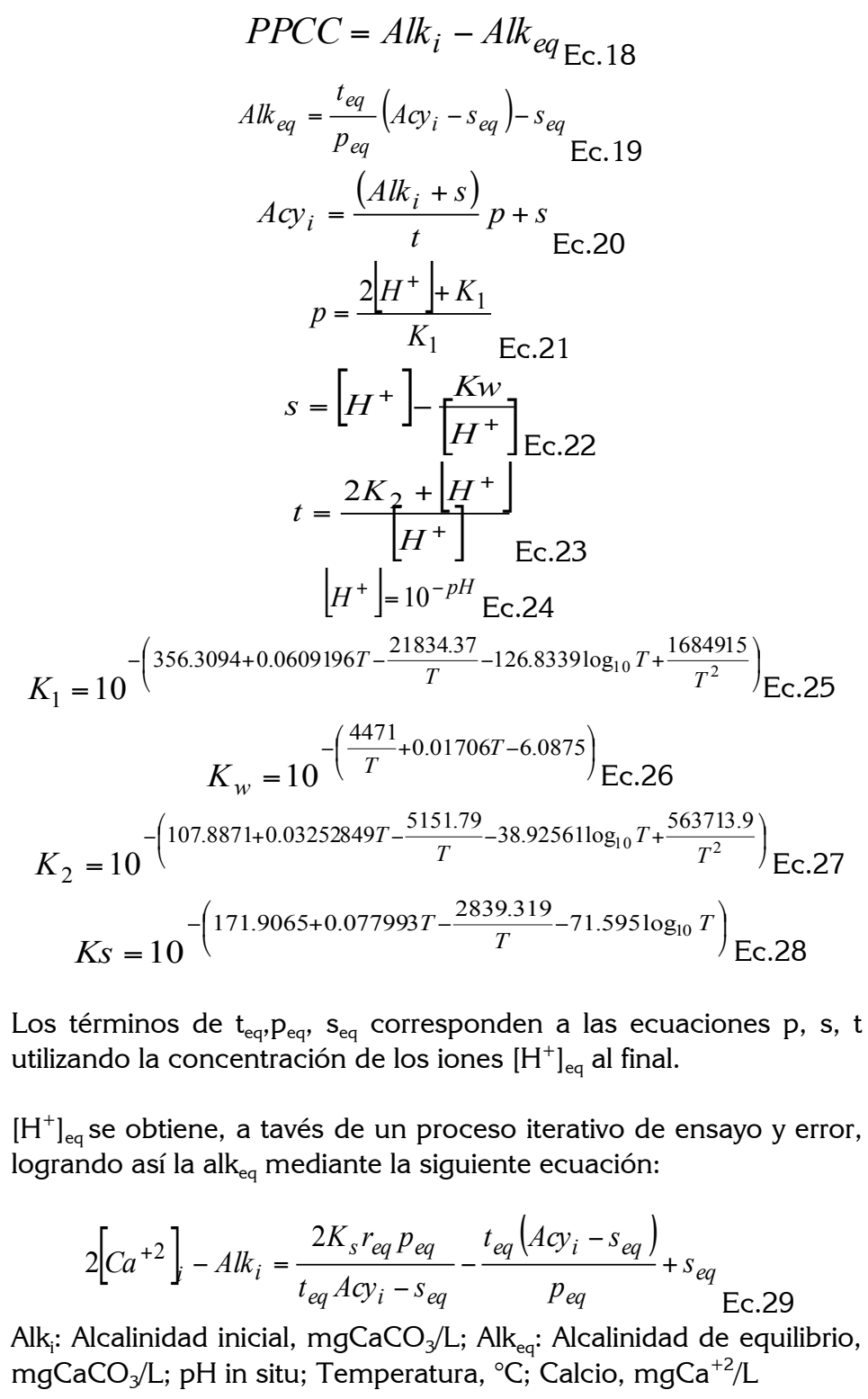 & $\begin{array}{l}\text { Utilidades: Indica la } \\
\text { masa teórica aproximada } \\
\text { del } \mathrm{CaCO}_{3} \text {, que podría } \\
\text { precipitar sobre la } \\
\text { superficie de una tubería. } \\
\text { Limitaciones: Dificultad } \\
\text { en la determinación de } \\
\text { las dosis químicas que se } \\
\text { aplican para obtener un } \\
\text { agua, con una PPCC } \\
\text { determinado. }\end{array}$ \\
\hline $\begin{array}{l}\text { Índice de } \\
\text { Agresividad (IA) }\end{array}$ & $A I=p H+\log (A * H)_{\text {Ec.30 }}$ & $\begin{array}{l}\text { Utilidades: Estima la } \\
\text { tendencia del agua a }\end{array}$ \\
\hline
\end{tabular}


Continuación tabla 1.

\begin{tabular}{|c|c|c|}
\hline ÍNDICE & ECUACIÓN & CARACTERÍSTICAS \\
\hline $\begin{array}{l}\text { IA }<10 \text {, } \\
\text { muy agresiva. } \\
10<\mathrm{IA}<12 \\
\text { moderadamente } \\
\text { agresiva. } \\
\text { IA }>12 \text {, no } \\
\text { agresiva. }\end{array}$ & $\begin{array}{l}\text { pH: Potencial de ión hidrógeno; A: Alcalinidad total, } \mathrm{mgCaCO}_{3} / \mathrm{L} \text {; } \\
\mathrm{H} \text { : Dureza cálcica, } \mathrm{mgCaCO}_{3} / \mathrm{L}\end{array}$ & $\begin{array}{l}\text { deteriorar la estructura de } \\
\text { la tubería. } \\
\text { Se aplica en tuberías de } \\
\text { asbesto cemento. } \\
\text { Limitaciones: No es } \\
\text { indicador de tendencia } \\
\text { para precipitar el } \mathrm{CaCO}_{3} \text {. }\end{array}$ \\
\hline $\begin{array}{l}\text { Relación de } \\
\text { Larson (RL) } \\
\text { Concentraciones } \\
\text { (mol/L), los } \\
\text { resultados } \\
\text { representados en } \\
\text { potencial de } \\
\text { corrosión: } \\
<0.2 \text {, No existe. } \\
0.2-0.4 \text {, } \\
\text { pequeño. } \\
0.4-0.5, \text { ligero. } \\
0.5-1.0, \\
\text { promedio. } \\
>1 \text {, Fuerte. }\end{array}$ & 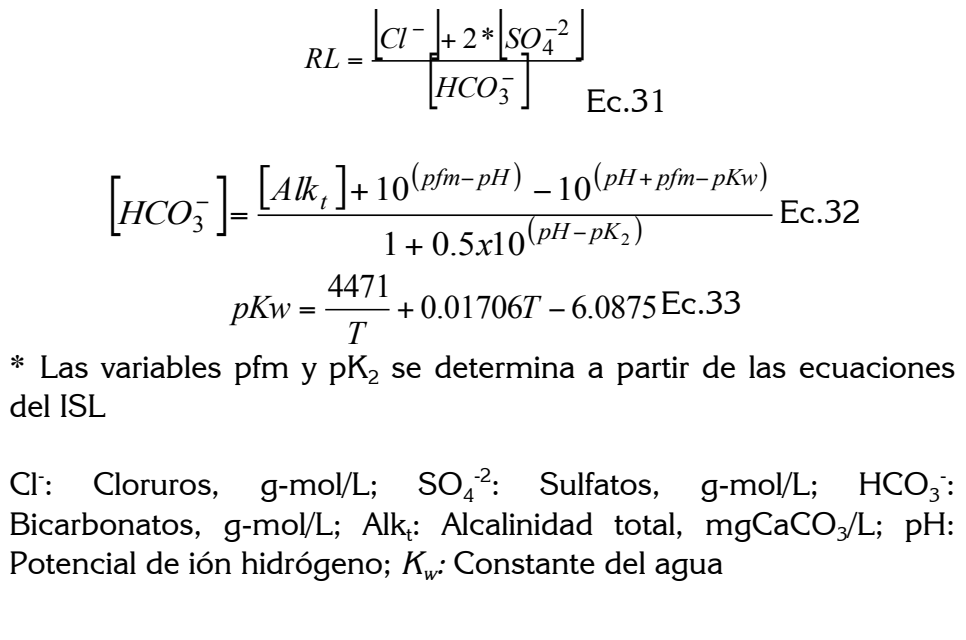 & $\begin{array}{l}\text { Utilidades: Representa } \\
\text { cuantitativamente la } \\
\text { corrosividad del agua, } \\
\text { debido a los iones } \\
\text { cloruro y sulfato. } \\
\text { Se aplica en tuberías } \\
\text { metálicas. } \\
\text { Limitaciones: Se } \\
\text { recomienda ser utilizado } \\
\text { para aguas con media a } \\
\text { alta alcalinidad (>50 } \\
\text { mgCaCO } \mathrm{CO}_{3} / \mathrm{L} \text { ). }\end{array}$ \\
\hline $\begin{array}{l}\text { Índice de } \\
\text { Estabilidad de } \\
\text { Ryznar (IER) } \\
\text { IER }<6.5 \text {, } \\
\text { incrustante. } \\
6.5<\text { IER }>7 \text {, } \\
\text { cercana al } \\
\text { equilibrio. } \\
\text { IER }>7 \text {, } \\
\text { corrosiva. }\end{array}$ & $\begin{array}{l}\qquad I E R=2 p H_{s}-p H_{\text {Ec. } 34} \\
\text { Se aplican las mismas ecuaciones del ISL } \\
\text { Alcalinidad total, } \mathrm{mgCaCO}_{3} / \mathrm{L} \text {; Dureza cálcica, } \mathrm{mgCaCO}_{3} / \mathrm{L} \text {; Sólidos } \\
\text { disueltos totales, } \mathrm{mg} / \mathrm{L} ; \mathrm{Con}_{\text {ductividad eléctrica, } \mu \mathrm{S} / \mathrm{cm} ; \mathrm{pH} \text { en el }} \\
\text { sitio; Temperatura en el sitio. }\end{array}$ & $\begin{array}{l}\text { Utilidades: Determina el } \\
\text { carácter corrosivo o } \\
\text { incrustante de las aguas. } \\
\text { Se aplica en tuberías de } \\
\text { acero. } \\
\text { Limitaciones: Tiende a } \\
\text { favorecer aguas de } \\
\text { mayor dureza y } \\
\text { alcalinidad total. } \\
\text { No ofrece ventajas } \\
\text { tangibles comparadas } \\
\text { con otros índices, para } \\
\text { calcular el estado de } \\
\text { saturación y potencial de } \\
\text { deposición del CaCO } \mathrm{CO}_{3}\end{array}$ \\
\hline $\begin{array}{l}\text { Relación de } \\
\text { Larson } \\
\text { Modificado } \\
\text { (RLM) } \\
\text { Un valor por } \\
\text { debajo de } 0,5 \text { no } \\
\text { son corrosivas y } \\
\text { aquellos con un } \\
\text { valor por encima } \\
\text { son corrosivos. }\end{array}$ & $\begin{array}{l}\qquad R L M=\frac{\left(\mathrm{Cl}^{-}+\mathrm{SO}_{4}^{-2}+\mathrm{Na}^{+}\right)^{1 / 2}}{\text { Alcalinidad }}\left(\frac{T}{25}\right) T R H \text { Ec.35 } \\
\mathrm{Cl}^{-} \text {: Concentración de cloruros, } \mathrm{mgCaCO}_{3} / \mathrm{L} ; \mathrm{SO}_{4}^{-2} \text { : Concentración } \\
\text { de sulfatos, } \mathrm{mgCaCO}_{3} / \mathrm{L} ; \mathrm{Na}^{+}: \mathrm{Concentración} \mathrm{de} \mathrm{sodio,} \\
\mathrm{mgCaCO}_{3} / \mathrm{L} ; \mathrm{T} \text { : Temperatura, }{ }^{\circ} \mathrm{C} ; \mathrm{TRH}: \text { Tiempo de retención } \\
\text { hidráulico, Días. }\end{array}$ & $\begin{array}{l}\text { Utilidades: Contiene } \\
\text { factores que influyen en } \\
\text { la corrosión (alcalinidad } \\
\text { total, pH y edad del } \\
\text { agua-TRH). } \\
\text { Evalúa el potencial de } \\
\text { corrosión. } \\
\text { Limitaciones: El término } \\
\text { de corrosión, en este } \\
\text { contexto, se refiere a la } \\
\text { tendencia a causar }\end{array}$ \\
\hline
\end{tabular}


Continuación tabla 1.

\begin{tabular}{|c|c|c|}
\hline ÍNDICE & ECUACIÓN & CARACTERÍSTICAS \\
\hline & & $\begin{array}{l}\text { problemas de agua de } \\
\text { color. } \\
\text { Su aplicación es } \\
\text { directamente sobre el } \\
\text { SDA. }\end{array}$ \\
\hline $\begin{array}{l}\text { Índice de } \\
\text { Corrosión de } \\
\text { Riddick (ICR) } \\
\text { ICR }<25 \text {, no es } \\
\text { corrosivo. } \\
26<\text { ICR }<50 \text {, } \\
\text { moderadamente } \\
\text { corrosivo. } \\
51<\text { ICR }<75 \text {, son } \\
\text { corrosivos. } \\
\text { ICR }>75 \text {, son muy } \\
\text { corrosivos. }\end{array}$ & 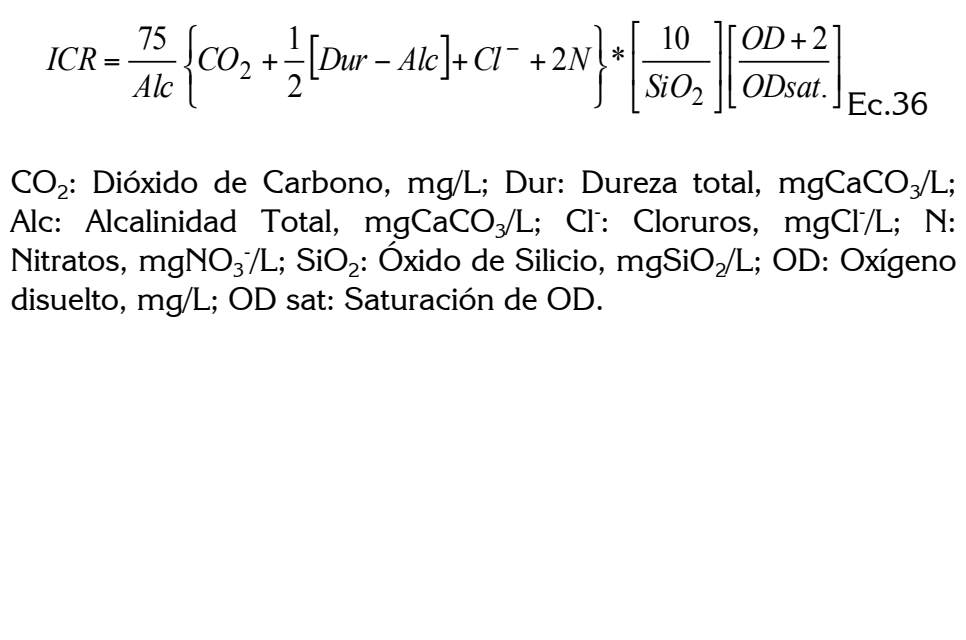 & $\begin{array}{l}\text { Utilidades: Introduce } \\
\text { factores, como OD, ion } \\
\mathrm{Cl}^{-} \text {y dureza total. } \\
\text { Intenta predecir la } \\
\text { cantidad de } \mathrm{CaCO}_{3} \text { que } \\
\text { se precipitará. } \\
\text { Limitaciones: Se aplica } \\
\text { para aguas blandas. } \\
\text { Requiere análisis } \\
\text { específicos, como } \\
\text { silicatos y saturación de } \\
\text { OD. } \\
\text { Su aplicación es } \\
\text { directamente sobre el } \\
\text { SDA. }\end{array}$ \\
\hline $\begin{array}{l}\text { Diagramas de } \\
\text { Caldwell- } \\
\text { Lawrence } \\
\text { Modificados }\end{array}$ & $\begin{array}{l}\text { Método gráfico, que requiere, como datos de entrada: } \mathrm{pH} \text {; dureza } \\
\text { cálcica, } \mathrm{mgCaCO}_{3} / \mathrm{L} ; \text { Magnesio, } \mathrm{mgCaCO}_{3} / \mathrm{L} ; \text { Alcalinidad Total, } \\
\mathrm{mgCaCO}_{3} / \mathrm{L} \text {; temperatura, }{ }^{\circ} \mathrm{C} \text {; fuerza iónica, para cuyo cálculo se } \\
\text { utilizan las ecuaciones aplicadas en el ISL. }\end{array}$ & $\begin{array}{l}\text { Utilidades: Determina el } \\
\text { equilibrio de saturación } \\
\text { del } \mathrm{CaCO}_{3} \text { y estima las } \\
\text { dosis químicas de } \\
\text { alcalinizantes. } \\
\text { Limitaciones: } \\
\text { Metodología de ensayo y } \\
\text { error, a menudo tedioso. } \\
\text { Los resultados se deben } \\
\text { verificar en campo. }\end{array}$ \\
\hline $\begin{array}{c}\text { Método de } \\
\text { Mojmir-Mach }\end{array}$ & $\begin{array}{l}\text { Empleando herramientas de informática, se fundamenta en el ISL y } \\
\text { en el gráfico de Tillmans; incorpora la fuerza iónica y considera la } \\
\text { aportación de los denominados iones ajenos (cationes y aniones } \\
\text { presentes en el agua diferentes al calcio, bicarbonatos, carbonatos } \\
\text { y protones). } \\
\text { El sistema de coordenadas representa las concentraciones } \\
\text { determinadas de }\left[\mathrm{H}_{2} \mathrm{CO}_{3}\right] \text { y }\left[\mathrm{HCO}_{3}^{-}\right] \text {, obtenidas a partir de la acidez } \\
\text { y la alcalinidad del agua, respectivamente, incluyendo los iones } \\
\text { ajenos en el equilibrio de saturación. }\end{array}$ & $\begin{array}{l}\text { Utilidades: Determina la } \\
\text { agresividad de un agua } \\
\text { mediante la acidez y } \\
\text { alcalinidad total. } \\
\text { Determina la } \\
\text { concentración de } \\
\text { hidróxido o } \mathrm{CaCO}_{3} \text { por } \\
\text { litro, que se requiere } \\
\text { adicionar para obtener } \\
\text { un agua en equilibrio } \\
\text { químico. } \\
\text { Limitaciones: Requiere } \\
\text { de software } \\
\text { especializado. } \\
\text { Los resultados se deben } \\
\text { verificar en campo. }\end{array}$ \\
\hline
\end{tabular}

Fuente: Singley, 1981; RAS, 2000; McNeill \& Edwards, 2001; Benefield \& Morgan, 2002; Schock, 2002; Varó Galváñ et al. 2004; APHA et al. 2005; Imran et al. 2005; Trujillo et al. 2008; De Sousa et al. 2010. 
para los problemas de corrosión (McNeill \& Edwards 2001; Imran et al. 2005), debido a que éste no tiene en cuenta la influencia de otros factores que aportan corrosión, como los iones cloruro y sulfato, la concentración de oxígeno disuelto, la velocidad de flujo, entre otros (Loewenthal et al. 2004).

Otra limitación de estos IE es que no predicen la cantidad de $\mathrm{CaCO}_{3}$ que precipitará en el SDA, por lo que autores, como Imran et al. (2005) y Birnhack et al. (2011), recomiendan que su aplicación sea acompañada con el índice PPCC, que representa la cantidad teórica aproximada del $\mathrm{CaCO}_{3}$ que podría precipitar sobre la superficie de una tubería. Otros autores, como Merrill \& Sanks (1977) y Loewenthal et al. (2004), sugieren valores de PPCC entre $4-10 \mathrm{mg} / \mathrm{L}$; sin embargo, cada SDA posee características propias y, por lo tanto, los valores adecuados se deben obtener realizando ensayos in situ.

Algunos de los IE tienen aplicación para tipos específicos de materiales de la tubería del SDA, tales como el IA, RL e IER. El IA, se basa en el pH y la solubilidad del carbonato de calcio, considerado una forma simplificada del ISL, siendo una herramienta de gran utilidad en la selección de materiales del SDA o en las opciones de tratamiento, para el control de la corrosión; no obstante, se debe tener en cuenta que se utiliza como medida de tendencia del agua a deteriorar la estructura de tuberías de asbesto cemento (Singley, 1981; Schock, 2002; Imran et al. 2005; Trujillo et al. 2008).

Los índices RL e IER son utilizados para aguas con mayor concentración de dureza y de alcalinidad (Schock, 2002; Trujillo et al. 2008); el RL, se desarrolló a partir del comportamiento corrosivo que aportan los cloruros y los sulfatos, con respecto a las propiedades protectoras del bicarbonato, siendo aplicable para tuberías metálicas (Schock, 2002;
Imran et al. 2005) y, el IER, es comúnmente utilizado, pero no ofrece ventajas tangibles comparadas con otros métodos, para calcular el estado de saturación del carbonato de calcio y su potencial de deposición (Schock, 2002), siendo, además, utilizado para tuberías de acero (Singley, 1981; APHA et al. 2005; Imran et al. 2005; Trujillo et al. 2008; De Sousa et al. 2010).

Los anteriores IE, se pueden emplear directamente en las plantas de potabilización, para evaluar el proceso de ajuste del pH; otros IE, como la RLM y el ICR, muestran el efecto del proceso directamente en el SDA, ya que consideran otras variables que dependen, tanto del equilibrio químico del agua como del transporte de la misma (tiempo de retención hidráulico, $\mathrm{CO}_{2}$, sodio, nitratos, $\mathrm{OD}$ y silicatos, entre otras) (Imran et al. 2005; Trujillo et al. 2008; De Sousa et al. 2010).

Los métodos gráficos, como los diagramas de Caldwell-Laurence modificados y Mojmir-Mach, determinan el equilibrio de saturación del $\mathrm{CaCO}_{3}$ y la agresividad del agua; estos métodos permiten determinar las dosis de alcalinizante requeridas para generar aguas con tendencia a precipitar $\mathrm{CaCO}_{3}$ (Merrill \& Sanks, 1977; RAS, 2000); sin embargo, se requiere un software especializado para obtener resultados, en corto tiempo, y verificarlos con aplicaciones prácticas (Benefield \& Morgan, 2002; Trujillo et al. 2008).

De acuerdo con el análisis anterior, las características del SDA de la ciudad de Santiago de Cali y la información disponible, los índices que fueron seleccionados para ser determinados con los datos históricos y las jornadas de muestreo fueron: Índice de saturación de Langelier-ISL, Índice de fuerza de conducción de Mc Cauley-IFC, Potencial de Precipitación de Carbonato de Calcio-PPCC, Índice de agresividad-IA, relación de Larson-RL e Índice de estabilidad de Ryznar-IER.

Tabla 2. Resumen estadístico de indicadores de tendencia central para las variables del proceso y determinación de índices.

\begin{tabular}{|c|c|c|c|c|c|c|c|c|}
\hline Variable & Media & Mín. & Máx. & $\mathbf{Q}_{1}$ & Med. & $Q_{3}$ & Valor recomendado & Fuente \\
\hline \multirow{4}{*}{$\begin{array}{c}\mathrm{pH} \\
\text { (unidades) }\end{array}$} & \multicolumn{6}{|c|}{ Históricos 2010 - 2011} & \multirow{4}{*}{$\begin{array}{l}\text { *Tub. Hierro: 6,8 - 7,3 } \\
\text { *Tub. Plomo y Cobre: } \\
8,0 \text { - 8,5 } \\
\text { *Tub. Latón (Aleación } \\
\text { Cobre y Zinc): < } 8.3 \\
\text { *Tub. Hormigón y Cinc: } \\
>8,5\end{array}$} & \multirow[b]{4}{*}{ WHO, 2011} \\
\hline & - & 6,50 & 8,20 & 6,94 & 7,17 & 7,46 & & \\
\hline & \multicolumn{6}{|c|}{ Monitoreos 2013} & & \\
\hline & - & 6,63 & 8,11 & 7,01 & 7,54 & 7,66 & & \\
\hline
\end{tabular}


Continuación tabla 2.

\begin{tabular}{|c|c|c|c|c|c|c|c|c|}
\hline Variable & Media & Mín. & Máx. & $\mathbf{Q}_{1}$ & Med. & $Q_{3}$ & Valor recomendado & Fuente \\
\hline \multirow{4}{*}{$\begin{array}{l}\text { Alcalinidad } \\
\text { Total } \\
\left(\mathrm{mgCaCO}_{3} / \mathrm{L}\right)\end{array}$} & \multicolumn{6}{|c|}{ Históricos 2010 - 2011} & \multirow{4}{*}{$\begin{array}{l}\text { Alcalinidad > } 40 \text { para la } \\
\text { formación de la película } \\
\text { de carbonato de calcio }\end{array}$} & \multirow{4}{*}{$\begin{array}{c}\text { Merrill \& } \\
\text { Sanks, } 1977\end{array}$} \\
\hline & 21,08 & 11,17 & 34,61 & 18,6 & 21,02 & 24,01 & & \\
\hline & \multicolumn{6}{|c|}{ Monitoreos 2013} & & \\
\hline & 21,47 & 11,90 & 29,40 & 19,73 & 21,40 & 22,83 & & \\
\hline \multirow{4}{*}{$\begin{array}{l}\text { Dureza Total } \\
\left(\mathrm{mgCaCO}_{3} / \mathrm{L}\right)\end{array}$} & \multicolumn{6}{|c|}{ Históricos 2010 - 2011} & \multirow{4}{*}{$40-60$} & \multirow{4}{*}{ RAS, 2000} \\
\hline & 45,76 & 19,73 & 71,37 & 19,73 & 45,55 & 51,88 & & \\
\hline & \multicolumn{6}{|c|}{ Monitoreos 2013} & & \\
\hline & 46,76 & 35,40 & 57,00 & 44,73 & 46,75 & 49,70 & & \\
\hline \multirow{4}{*}{$\begin{array}{c}\text { Dureza Cálcica } \\
\left(\mathrm{mgCaCO}_{3} / \mathrm{L}\right)\end{array}$} & \multicolumn{6}{|c|}{ Históricos 2010 - 2011} & \multirow{4}{*}{$\begin{array}{l}>40 \text { para la formación } \\
\text { de la película de car- } \\
\text { bonato de calcio }\end{array}$} & \multirow{8}{*}{$\begin{array}{c}\text { Merrill \& } \\
\text { Sanks, } 1977\end{array}$} \\
\hline & 33,14 & 8,01 & 28,65 & 28.,65 & 33,30 & 38,00 & & \\
\hline & \multicolumn{6}{|c|}{ Monitoreos 2013} & & \\
\hline & 32,46 & 17,60 & 38,80 & 30,15 & 33,45 & 34,75 & & \\
\hline \multirow{4}{*}{$\begin{array}{l}\text { Calcio } \\
(\mathrm{mg} / \mathrm{L})\end{array}$} & \multicolumn{6}{|c|}{ Históricos 2010 - 2011} & \multirow{4}{*}{$\begin{array}{l}>16 \text { para la formación } \\
\text { de la película de car- } \\
\text { bonato de calcio }\end{array}$} & \\
\hline & 13,29 & 3,21 & 21,66 & 11,49 & 13.35 & 15,20 & & \\
\hline & \multicolumn{6}{|c|}{ Monitoreos 2013} & & \\
\hline & 13,01 & 7,05 & 15,55 & 12,08 & 13,41 & 13,93 & & \\
\hline \multirow{4}{*}{$\begin{array}{l}\text { Magnesio } \\
\left(\mathrm{mgMg}^{+2} / \mathrm{L}\right)\end{array}$} & \multicolumn{6}{|c|}{ Históricos 2010 - 2011} & \multirow{4}{*}{$\begin{array}{l}\text { 3-100 para minimizar la } \\
\text { formación de hidróxido } \\
\text { de magnesio a tempera- } \\
\text { turas elevadas }\end{array}$} & \multirow{4}{*}{ RAS, 2000} \\
\hline & 3,06 & 0,46 & 6,15 & 2.28 & 3,00 & 3,77 & & \\
\hline & \multicolumn{6}{|c|}{ Monitoreos 2013} & & \\
\hline & 3,47 & 1,87 & 5,22 & 3,09 & 3,46 & 3,75 & & \\
\hline \multirow{4}{*}{$\begin{array}{c}\text { Temperatura } \\
\left({ }^{\circ} \mathrm{C}\right)\end{array}$} & \multicolumn{6}{|c|}{ Históricos 2010 - 2011} & Afecta parámetros & \\
\hline & - & 18,0 & 29,8 & 21,8 & 22,6 & 23,6 & como: solubilidad del & \\
\hline & Monito & 2013 & & & & & las propiedades del & $\begin{array}{l}\text { McNeill \& } \\
\text { Edwards }\end{array}$ \\
\hline & - & 22,70 & 25,70 & 23,43 & 23,95 & 24,45 & $\begin{array}{l}\text { agua (viscosidad y la } \\
\text { movilidad de iones), } \\
\text { tasa de lixiviación de } \\
\text { metales, etc. }\end{array}$ & 2001 \\
\hline & Históric & 2010 & 2011 & & & & & \\
\hline Cloruros & 15,74 & 2,97 & 27,45 & 12,48 & 16,23 & 18,42 & & \\
\hline (mg/L) & Monitol & ss 2013 & & & & & $>50$ cloruros o sulfatos & \\
\hline & 16,43 & 14,60 & 20,16 & 14,60 & 15,92 & 17,36 & son considerados como & Loewenthal \\
\hline & Históric & 2010 & 2011 & & & & potencialmente corro- & Gray, 2008 \\
\hline Sulfatos & 13,56 & 3,05 & 49,76 & 8,52 & 10,39 & 17,98 & & \\
\hline$(\mathrm{mg} / \mathrm{L})$ & Monito & 2013 & & & & & & \\
\hline & 12,47 & 10,00 & 14,90 & 11,33 & 12,55 & 13,55 & & \\
\hline & Históric & 2010 & 2011 & & & & Un incremento de con- & \\
\hline Conductividad & 127,4 & 96,9 & 212,0 & 96,9 & 126,7 & 134,6 & ductividad $>50 \%$ en el & \\
\hline (uc/sm) & Monito & 2013 & & & & & bio en la cantidad de & VDT, 2007 \\
\hline (20/(-111) & 143,4 & 129,9 & 151,5 & 138,7 & 145,5 & 147,9 & $\begin{array}{l}\text { SDT que incluyen los } \\
\text { cloruros y el sodio }\end{array}$ & \\
\hline
\end{tabular}


La tabla 2 muestra el resumen del análisis estadístico de indicadores de tendencia central de los registros históricos, suministrados por EMCALI EICE ESP, correspondiente al periodo 2010 - 2011 y los monitoreos de calidad del agua tratada del río Cauca.

Se observa que la alcalinidad total y dureza cálcica, presentan concentraciones menores de $40 \mathrm{mgCaCO}_{3} / \mathrm{L}$, valor recomendable para promover la formación de la película de carbonato de calcio en el SDA, lo que indica la necesidad de buscar estrategias para incrementar estos valores, ajustando el pH, de acuerdo a los materiales utilizados en cada SDA (Merrill \& Sanks, 1977; Varó Galváñ et al. 2004; WHO, 2011).

Aunque los cloruros y los sulfatos tienden a fomentar la corrosión mediante la formación de una película de óxido (Loewenthal et al. 2004), los niveles en el agua tratada del río Cauca son menores $50 \mathrm{mg} / \mathrm{L}$-valor que indica corrosividad, según Gray (2008)-. Este comportamiento, se relaciona con la conductividad, ya que representa una parte de los cloruros y al contener bajas concentraciones ( $<50 \mathrm{mgCl}^{-} / \mathrm{L}$ ), los niveles de conductividad no superan los 1000 microsiemens/ cm, establecido en la Resolución 2115 de 2007 (Imran et al. 2005; MPS \& MAVDT, 2007).

La tabla 3 muestra los resultados de la determinación de los IE seleccionados, encontrándose que, en general, el agua presenta condiciones insaturadas respecto al $\mathrm{CaCO}_{3}$ y con tendencia a la corrosión.

Según el ISL, el agua es insaturada (valores negativos), debido a la baja alcalinidad del agua (Tam \& Elefsiniotis, 2009; Wilczak et al. 2010). El máximo ISL determinado con los datos históricos $(0,24)$, estuvo relacionado con un agua tratada, con pH aproximado de 8.8 unidades, mientras el RAS (2000), reglamenta un ISL de 0.2. Varó Galváñ et al. (2004) sugieren un valor inferior a 0.5 e Imran et al. (2005) y De Sousa et al. (2010), entre 0,2 - 1,0.

El IFC también clasifica el agua como insaturada. Según Benefield \& Morgan (2002), los valores bajos de calcio y de carbonatos no permiten llegar al equilibrio ni mantener una capacidad buffer. Tanto el ISL como el IFC son útiles para proporcionar las condiciones de saturación respecto al $\mathrm{CaCO}_{3}$; sin embargo, no predicen la cantidad de $\mathrm{CaCO}_{3}$ que se precipitará en el SDA, siendo recomendable su uso, de manera complementaria con el PPCC (Imran et al. 2005; Birnhack et al. 2011).

Los valores negativos del PPCC indican que el $\mathrm{CaCO}_{3}$ no tiende a precipitarse; el valor máximo presentado $(0.2)$ correspondió a un agua con alcalinidad de $32,3 \mathrm{mgCaCO} / \mathrm{L}$, la cual, se encuentra dentro del rango para la alcalinidad de equilibrio (Alkeq: 27,3 - 36,7 mg СаСО $/ \mathrm{L}$ ), siendo este mismo valor el que presentó un ISL positivo. Merrill \& Sanks (1977) y Loewenthal et al. (2004) recomiendan que se deben generar valores de PPCC entre 4-10 mg/L, lo que se lograría si el agua tratada del río Cauca alcanzara una alcalinidad promedio entre $34-44 \mathrm{mg} \mathrm{CaCO} / \mathrm{L}$.

El IA presentó valores cercanos a 10,0, lo que permite clasificar el agua como moderadamente agresiva (10 - 12), según Tam \& Elefsiniotis, (2009), debido a la baja alcalinidad al final de la potabilización.

Con base en la RL, Trujillo et al. (2008) y Agatemor \& Okolo (2008) muestran que el bajo nivel de bicarbonatos, en relación con los cloruros y sulfatos, es un factor importante, que influye en la tendencia corrosiva del agua, sugiriéndose un RL entre 0,2 - 0,3 (expresando las variables en $\mathrm{mol} / \mathrm{L}$ ), promoviendo características de alcalinidad total y dureza cálcica, de 30,5 mgCaCO $3 / \mathrm{L}$ (Melidis et al. 2007). El agua tratada del río Cauca, por presentar concentraciones de alcalinidad por debajo de $40 \mathrm{mgCaCO}_{3} / \mathrm{L}$, registró valores promedio de RL, entre históricos y monitoreos, de casi el doble del límite que clasifica el agua con fuerte potencial de corrosión para las tuberías metálicas. Por lo tanto, teniendo en cuenta que una de las limitaciones de este índice son los valores de alcalinidad, se sugiere no aplicarlo para aguas, como las del agua tratada del río Cauca.

Según el IER, el agua es corrosiva para las tuberías de acero; sin embargo, debido a que este material representa sólo el $1,2 \%$ del SDA de Santiago de Cali, se sugiere no emplearlo; además, de que no es aplicable a aguas con baja alcalinidad (De Sousa et al. 2010). Autores, como Varó Galváñ et al. (2004) indican que aún no existe un índice de estabilización de $\mathrm{pH}$ que sea aplicable universalmente, así como Imran et al. (2005) y De Sousa et al. (2010), quienes plantean que no se ha desarrollado un IE que represente el comportamiento global del agua en un SDA. En el caso del SDA de la ciudad de Santiago de Cali, los ISL, IFC, PPCC, IA, RL e IER pueden ser empleados en la planta de potabilización evaluada; no obstante, la determinación de PPCC requiere un proceso iterativo de ensayo y error, para obtener las condiciones de equilibrio al no contar con la aplicación de un software especializado.

El ISL y el IFC tienen una relación tenue con las tasas reales de corrosión en los SDA, pero aún no se descarta su importancia en el proceso de ajuste del $\mathrm{pH}$, ya que son indicadores de saturación del agua respecto al $\mathrm{CaCO}_{3}$ que, al mantener un cambio sostenido en el $\mathrm{pH}$, tendría un impacto definitivo en la resistencia a la corrosión de las tuberías viejas (Imran et al. 2005), pero no se deben utilizar para ningún otro propósito. 
Tabla 3. Comportamiento de Índices de estabilización para el agua tratada del río Cauca.

\begin{tabular}{|c|c|c|c|c|c|c|c|}
\hline \multirow{2}{*}{ Índice } & \multicolumn{6}{|c|}{ Indicadores de tendencia central } & \multirow{2}{*}{ Interpretación } \\
\hline & Media & Mín. & Máx. & $Q_{1}$ & Mediana & $Q_{3}$ & \\
\hline \multirow{4}{*}{ ISL } & \multicolumn{6}{|c|}{ Históricos 2010 - 2011} & \multirow{4}{*}{$\begin{array}{l}\text { Agua insaturada } \\
\text { con } \mathrm{CaCO}_{3}\end{array}$} \\
\hline & $-1,7$ & $-3,8$ & 0,24 & $-2,0$ & $-1,7$ & $-1,4$ & \\
\hline & \multicolumn{6}{|c|}{ Monitoreos 2013} & \\
\hline & $-1,4$ & $-2,4$ & $-0,7$ & $-1,8$ & $-1,2$ & $-1,1$ & \\
\hline \multirow{4}{*}{ IFC } & \multicolumn{6}{|c|}{ Históricos $2010-2011$} & \multirow{4}{*}{$\begin{array}{l}\text { Agua insaturada } \\
\text { con tendencia a } \\
\text { disolver } \mathrm{CaCO}_{3}\end{array}$} \\
\hline & 0,01304 & 0,00007 & 0,10131 & 0,00470 & 0,00802 & 0,01682 & \\
\hline & \multicolumn{6}{|c|}{ Monitoreos 2013} & \\
\hline & 0,05133 & 0,00177 & 0,16942 & 0,00983 & 0,03986 & 0,08640 & \\
\hline \multirow{4}{*}{ PPCC } & \multicolumn{6}{|c|}{ Históricos 2010 - 2011} & \multirow{4}{*}{$\begin{array}{c}\text { No precipita } \\
\mathrm{CaCO}_{3}\end{array}$} \\
\hline & $-12,3$ & $-73,4$ & 0,2 & $-13,6$ & $-9,6$ & $-7,0$ & \\
\hline & \multicolumn{6}{|c|}{ Monitoreos 2013} & \\
\hline & $-8,6$ & $-20,1$ & $-3,4$ & $-12,3$ & $-6,3$ & $-5,2$ & \\
\hline \multirow{4}{*}{ IA } & \multicolumn{6}{|c|}{ Históricos $2010-2011$} & \multirow{4}{*}{$\begin{array}{l}\text { Agua mod- } \\
\text { eradamente } \\
\text { agresiva }\end{array}$} \\
\hline & 10,0 & 7,9 & 11,9 & 9,7 & 9,9 & 10,3 & \\
\hline & \multicolumn{6}{|c|}{ Monitoreos 2013} & \\
\hline & 10,3 & 9,3 & 11,0 & 9,9 & 10,4 & 10,6 & \\
\hline \multirow{4}{*}{$\mathrm{RL}$} & \multicolumn{6}{|c|}{ Históricos 2010 - 2011} & \multirow{4}{*}{$\begin{array}{l}\text { Agua con fuerte } \\
\text { potencial de } \\
\text { corrosión }\end{array}$} \\
\hline & 1,8 & 0,8 & 4,9 & 1,4 & 1,7 & 2,1 & \\
\hline & \multicolumn{6}{|c|}{ Monitoreos 2013} & \\
\hline & 1,9 & 1,2 & 2,9 & 1,5 & 1,8 & 2,3 & \\
\hline \multirow{4}{*}{ IER } & Históricos & $2010-20$ & & & & & \multirow{4}{*}{ Agua corrosiva } \\
\hline & 10,6 & 8,3 & 13,6 & 10,2 & 10,6 & 10,8 & \\
\hline & \multicolumn{6}{|c|}{ Monitoreos 2013} & \\
\hline & 10,3 & 9,5 & 11,7 & 9,8 & 10,0 & 10,7 & \\
\hline
\end{tabular}

El IA indica las condiciones del agua en las tuberías de AC; este material es uno de los de mayor aplicación en los SDA y ocasionan mayores daños, debido a las características químicas del agua, como aguas blandas y los suelos donde se instalan (Hu \& Hubble, 2007; Pérez-Vidal et al. 2012). En el caso del SDA de la ciudad de Santiago de Cali, está conformado por PVC (51,6\%), asbesto cemento - AC (31,9\%), hierro (fundido, dúctil, galvanizado) (11,5\%), cilindros de acero revestidos en concreto - CCP $(2,8 \%)$, acero $(1,2 \%)$ y Polietileno de alta densidad (1\%) y, adicionalmente, el tramo de tubería de $\mathrm{AC}$, es el que presenta el mayor número de daños.

El PVC es altamente resistente a agentes químicos, mientras que el AC y el hierro favorecen los procesos de lixiviación de metales, lo que indica la necesidad de ajustar el $\mathrm{pH}$ a valo- res mayores a 8,5 unidades, según lo recomendando por la WHO (2011), para tuberías de AC; este ajuste no causaría problemas de incrustación de $\mathrm{Mg}(\mathrm{OH})_{2}$ en altas temperaturas, ya que las concentraciones promedio de magnesio, en este tipo de agua $(3,06 \mathrm{mg} / \mathrm{L})$, se encuentran dentro del rango permitido (RAS, 2000).

Al propiciar mejores condiciones para la formación de la película de $\mathrm{CaCO}_{3}$ para el SDA de Santiago de Cali, se podrían aplicar los índices RL e IER, ya que, actualmente, no deben ser empleados por no ser recomendables para aguas con baja alcalinidad (Benefield \& Morgan 2002; Varó Galváñ et al. 2004). Los demás índices, como RLM e ICR, deberían ser incluidos en futuros análisis en el SDA, puesto que consideran otras variables que influyen en el transporte del agua, 
tales como el tiempo de retención hidráulico, $\mathrm{CO}_{2}$, sodio, nitratos, OD y silicatos (Imran et al. 2005; Trujillo et al. 2008; De Sousa et al. 2010).

Actualmente, el producto aplicado en el proceso de ajuste del $\mathrm{pH}$ es la cal viva que, posteriormente, se hidrata - $\mathrm{Ca}(\mathrm{OH})_{2}$; sin embargo, este producto presenta inconvenientes, como insolubilidad de algunas sales e incremento de la turbiedad en el agua tratada (Di Bernardo \& Sabogal Paz, 2008), por lo que se sugiere la evaluación de otros alcalinizantes, tales como hidróxido de sodio $(\mathrm{NaOH})$, carbonato de sodio $\left(\mathrm{Na}_{2} \mathrm{CO}_{3}\right)$, bicarbonato de sodio $\left(\mathrm{NaHCO}_{3}\right)$, entre otros, que se han utilizado para aguas con baja alcalinidad (Maddison et al. 2001), como el agua tratada del río Cauca; además de ajustar los valores de alcalinidad total del agua tratada del río Cauca, entre 37 - 43mg $\mathrm{CaCO}_{3} / \mathrm{L}$, para promover la formación de la película de carbonato de calcio en el SDA (Varó Galváñ et al. 2004; WHO, 2011).

Para el SDA de la ciudad de Santiago de Cali, se debe evaluar la posibilidad de continuar sustituyendo las tuberías de AC y hierro, que son materiales que favorecen la lixiviación de metales y causan el mayor número de daños. El PVC, que actualmente es el principal material del SDA, es altamente resistente a agentes químicos y propicio para la distribución del agua potable (Volk et al. 2000).

En general, este estudio permite concluir que la adecuada utilización de los índices de estabilización, como método indirecto de monitoreo desde las plantas de tratamiento, permite prever el comportamiento del agua en un SDA, valorando sus características agresivas, corrosivas o incrustantes. La aplicación de los índices de estabilización debe tener en cuenta su utilidad y sus limitaciones, para generar resultados confiables y acertados, siendo una alternativa económica y viable, que puede ser utilizada desde las plantas de tratamiento.

Este estudio permitió verificar que, efectivamente, la aplicación de un sólo índice no permite representar el comportamiento global del agua en un SDA, siendo recomendable utilizar, al menos, un índice, que proporcione información sobre la tendencia de precipitación de $\mathrm{CaCO}_{3}$ junto con el cálculo de la PPCC y otro que represente las necesidades del SDA, de acuerdo con el tipo de material de las tuberías.

La determinación de los diferentes índices permitió identificar que se deben mejorar las condiciones del ajuste del $\mathrm{pH}$ y que para esta agua no es recomendable usar los índices RL e IER, debido a los bajos niveles de alcalinidad. En caso de mejorarse esta condición en el proceso de ajuste del $\mathrm{pH}$, su determinación sería útil, para identificar problemas de corrosión o de incrustación en tuberías metálicas y de acero.
Como estrategias de mejoramiento del proceso de ajuste del pH y de prevención de problemas en el SDA, se recomienda evaluar otros alcalinizantes, establecer correlaciones entre variables de fácil y rápida medición para el control operacional del proceso y continuar con la renovación de tuberías en el SDA, por materiales como el PVC.

Agradecimientos: Los autores agradecen el apoyo de EMCALI EICE ESP, por el suministro de la información histórica y facilitar la toma de las muestras del agua tratada del río Cauca, en la Planta de Potabilización de Puerto Mallarino (Cali, Valle), a la Universidad del Valle y a Colciencias. Conflictos de intereses: Certificamos la originalidad y el carácter inédito del manuscrito preparado y revisado con la participación de todos los autores, quienes declaramos que no existe ningún conflicto de intereses, que ponga en riesgo la validez de los resultados. Financiación: Este estudio fue financiado por Colciencias - Programa de fomento a la formación de investigadores (Jóvenes Investigadores e innovadores) y la Universidad del Valle, mediante el Proyecto "Evaluación del proceso de estabilización del $\mathrm{pH}$ mediante la aplicación de índices y estabilizadores en el agua tratada del río Cauca”.

\section{BIBLIOGRAFÍA}

1. AGATEMOR, C.; OKOLO, P.O. 2008. Studies of corrosion tendency of drinking water in the distribution system at the University of Benin. J. Environmentalist (Netherlands). 28(4):379-384.

2. APHA; AWWA; WEF. 2005. Standard methods for the examination of water and wastewaters. 21st ed. Water Environment Federation (USA). 282p.

3. BENEFIELD, L.D.; MORGAN, J.M. 2002. Precipitación química. En: Letterman R. Calidad y Tratamiento del agua: Manual de suministros de agua comunitaria. $5^{a}$ ed. Ed. Mc Graw Hill (España). p.1048-1157.

4. BIRNHACK, L.; VOUTCHKOV, N.; LAHAV, O. 2011. Fundamental chemistry and engineering aspects of posttreatment processes for desalinated water - A review. J. Desalin. (Netherlands). 273(1):6-22.

5. CASEY, T.J. 2009. Water stabilisation and corrosion control. J. Aquavarra Res. R\&D Publications (Ireland). Paper 2:1-12.

6. DE SOUSA, C.; CORREIA, A.; COLMENARES, M.C. 2010. Corrosión e incrustaciones en los sistemas de distribución de agua potable: Revisión de las estrategias de control. Bol. Malariología y Salud Ambiental (Venezuela). 50(2):187-196. 
7. DI BERNARDO, L.; SABOGAL PAZ, L. 2008. Seleção de Tecnologias de Tratamento de Água. São Carlos: Editora LDIBE LTDA. Vol. I. 878p.

8. EDZWALD, J.K. 2010. Water Quality \& Treatment: A Handbook on Drinking Water, American Water Works Association. New York: McGraw Hill. 1696p.

9. FURTADO, P.S.; POERSCH, L.H.; WASIELESKY, W. 2011. Effect of calcium hydroxide, carbonate and sodium bicarbonate on water quality and zootechnical performance of shrimp Litopenaeus vannamei reared in bio-flocs technology (BFT) systems. J. Aquacult. (Netherlands). 321(1-2):130-135.

10. GRAY, N.F. 2008. Drinking Water Quality: Problems and Solutions. 2a Ed. Cambridge University Press (United States). 520p.

11. HU, Y.; HUBBLE, D.W. 2007. Factors contributing to the failure of asbestos cement water mains. Can. J. Civil Engineering. 34(5):608-621.

12. IMRAN, S.A.; DIETZ, J.D.; MUTOTI, G.; TAYLOR, J.S.; RANDALL, A.A. 2005. Modified Larsons Ratio incorporating temperature, water age, and electroneutrality effects on red water release. J. Environm. Eng. (United States). 131(11):1514-1520.

13. LOEWENTHAL, R.E.; MORRISON, I.; WENTZEL, M.C. 2004. Control of corrosion and aggression in drinking water systems. Water Sci. Technol. (England). 49(2):9-18.

14. MADDISON, L.A.; GAGNON, G.A.; EISNOR, J.D. 2001. Corrosion control strategies for the Halifax regional distribution system. Can. J. Civil Engineering. 28(2):305-313.

15. MCNEILL, L.S.; EDWARDS, M. 2001. Review of pipe corrosion in drinking water distribution systems. J. Am. Water Works Assoc. (United States). 93(7):88-100.

16. MELIDIS, P.; SANOZIDOU, M.; MANDUSA, A.; OUZOUNIS, K. 2007. Corrosion control by using indirect methods. J. Desalin. 213(1):152-158.

17. MERRILL, D.T.; SANKS, R.L. 1977. Corrosion control by deposition of $\mathrm{CaCO} 3$ Films: Part 1, A Practical Approach for Plant Operators. J. Am. Water Works Assoc. 69(11):592-599.

18. MPS - MINISTERIO DE PROTECCIÓN SOCIAL; MINISTERIO DE AMBIENTE, VIVIENDA Y DESARROLLO
TERRITORIAL - MAVDT. REPÚBLICA DE COLOMBIA, Resolución 2115 de 2007.

19. PÉREZ-VIDAL, A.; AMÉZQUITA-MARROQUÍN, C.P.; TORRES-LOZADA, P. 2012. Identificación y priorización de peligros como herramientas de la gestión del riesgo en sistemas de distribución de agua potable. Rev. Ingenier. Universidad. (Colombia). 16(2):449-469.

20. RAS - Reglamento Técnico del Sector de Agua Potable y Saneamiento Básico. 2000. Ministerio de Desarrollo Económico y Dirección de Agua Potable y Saneamiento Básico, Sección II. (Colombia). TíTULO C: Sistemas de Potabilización. 184p.

21. SCHOCK, M.R. 2002. Corrosión interna y control de deposición. En: Letterman R. Calidad y Tratamiento del agua: Manual de suministros de agua comunitaria. $5^{\mathrm{a}}$ ed. Ed. Mc Graw Hill (España). 1048-1157p.

22. SINGLEY, J.E. 1981. The search for a corrosion index. J. Am. Water Works Assoc. 73(11):579-582.

23. TAM, Y.S.; ELEFSINIOTIS, P. 2009. Corrosion control in water supply systems: Effect of $\mathrm{pH}$, alkalinity, and orthophosphate on lead and copper leaching from brass plumbing. J. Environ. Sci. Health A (UK). 44(12):1251-1260.

24. TREJO VÁZQUEZ, R.; GARCÍA DÍAZ, L. 2007. Modelación de la Deriva del pH en Función del Tiempo en Muestras de Agua Potable. Rev. Conciencia Tecnológica (México). 33:12-16.

25. TRUJILLO, E.; MARTÍNEZ, V.; FLORES, N.S. 2008. Ajuste del Equilibrio Químico del Agua Potable con Tendencia Corrosiva por Dióxido de Carbono. Rev. Información Tecnológica (Chile). 19(6):89-101.

26. VARÓ GALVÁÑ, P.; CHILLÓN, M.F.; PRATS, D. 2004. Características Fisicoquímicas de las Aguas Ablandadas. Ingeniería Química. Universidad de Alicante. 146-150p. Disponible desde Internet en http://www. inese.es/html/files/pdf/amb/iq/409/09ARTICULOEN. pdf nlc-bnc.ca/iso/tc46sc9/standard/690-2e.htm (con acceso el 20/10/2013).

27. VOLK, C.; DUNDORE, E.; SCHIERMANN, J.; LECHEVALLIER, M. 2000. Practical evaluation of iron corrosion control in a drinking water distribution system. J. Water Res. (England). 34(6):1967-1974. 
28. WHO - World Health Organization. 2011. Guidelines for drinking - water quality. 4th edition. Geneva, Switzerland: WHO Press. 564p.
29. WILCZAK, A.; HOKANSON, D.R.; TRUSSELL, R.R.; BOOZARPOUR, M.; DEGRACA, A.F. 2010. Water conditioning for LCR compliance and control of metals release in San Francisco's water system. J. Am. Water Works Assoc. 102(3):52-64.

Recibido: Febrero 27 de 2014

Aceptado: Agosto 27 de 2014

\section{Como citar:}

Bueno-Zabala, K.A.; Torres-Lozada, P.; Delgado-Cabrera, L.G. 2014. Monitoreo y medición del ajuste del pH del agua tratada del río Cauca mediante índices de estabilización. Rev. U.D.C.A Act. \& Div. Cient. 17(2): 563-575. 